\title{
A DISCRETE-TIME ROBUST ADAPTIVE PI CONTROLLER FOR GRID-CONNECTED VOLTAGE SOURCE CONVERTER WITH LCL FILTER
}

\author{
Paulo J. D. O. Evald ${ }^{1,2}$, Guilherme V. Hollweg ${ }^{1}$, Rodrigo V. Tambara ${ }^{1}$, Hilton A. Gründling ${ }^{1}$ \\ ${ }^{1}$ Federal University of Santa Maria, Santa Maria - RS, Brazil \\ ${ }^{2}$ Franciscan University, Santa Maria - RS, Brazil \\ e-mail: paulo.evald@gmail.com,guilherme.hollweg@gmail.com,rodvarella@yahoo.com.br,ghilton03@gmail.com
}

\begin{abstract}
In this work, it is presented a new direct discrete-time robust adaptive PI (Proportional Integral) Controller for grid-injected current control loop of a voltage source converter with LCL filter. The mathematical background is based on Robust Model Reference Adaptive Control theory. However, the proposed controller is straightforward, it does not need a reference model and has capability to track directly currents reference. This approach simplifies significantly controller design, resulting in a reformulation of parameters vector used for adaptation of adjustable gains. It turns the controller robust to unmodelled dynamics, while avoid the complexity inherent to the conventional high order adaptive controllers for gridconnected power systems. Besides, it is highlighted that proposed controller does not need resonant controllers for grid disturbance rejection, or require any knowledge of grid parameters, lines impedance or load power demand. Also, due to its simple structure, it is easily implemented and does not require a high processing capacity. Furthermore, the effectiveness of the control strategy in terms of reference tracking, harmonics content and robustness to the grid impedance variation is corroborated through experiments.
\end{abstract}

Keywords - Computational Burden Reduction, Discretetime Controller, Grid-tied Converters, LCL Filter, Robust Adaptive PI Controller.

\section{INTRODUCTION}

Since the beginning of Second Industrial Revolution, the demand for electrical energy has increasing significantly [1], supplied by conventional fuel technologies [2]. However, natural resources reserves, such as coal and oil, are limited and have fast been ending, being necessary adhere to alternative power generation forms.

As renewable energy systems can reduce relevantly the dependence on fossil fuels for electricity generation [3]-[5] and decreases carbon emissions, nowadays there is a great demand for renewable energy generation [6]. Due to the multi-benefits of these systems [7], [8], there is an increasing investment on clean energy, such as solar, wind and others renewable sources [9]. It is achieved thanks to power electronics technologies development, which raises efficiency of renewable energy generation [10], [11].

Manuscript received 09/07/2020; first revision 11/10/2020; accepted for publication 12/10/2020, by recommendation of Editor Demercil de Souza Oliveira Jr. http://dx.doi.org/10.18618/REP.2021.1.0053
Generally, VSC (Voltage Source Converters) are used to integrate the clean energy source to the electrical grid [12], with AC filters as interface. These filters are employed to reduce THD (Total Harmonic Distortion), produced by converter switching [13], once the quality of power is always a concern for the high penetration of grid-connected power systems [14]. It guarantees that power injection is in compliance with specific grid-connected standards [15], such as IEEE 1547 [16], standard for interconnecting distributed resources with electric power systems. Among the AC filters, $\mathrm{L}$ and LCL configurations are the two most used solutions, where the LCL filter presents a set of advantages, such as: higher attenuation $(-60 \mathrm{~dB} / \mathrm{dec}$ in face of $-20 \mathrm{~dB} / \mathrm{dec}$ of $\mathrm{L}$ filter) and smaller reactive components, which implies in a lower cost, weight, switching frequency and reactive power consumption in the fundamental frequency of the grid [17].

In this bias, several control laws for internal LCL current loop control and external voltage loop control of the gridconnected VSC have been proposed. The internal loop is used to ensure that injected currents are in phase with grid voltage, with proper amplitude, providing active power. Meanwhile, the external loop is used to voltage source control. For internal current loop control, which is the main focus of the investigation in this work, stand out the following approaches: PI (Proportional-Integral) Controller [13], PR (Proportional-Resonant) Controller [18], Deadbeat Controller [19], Feedback Controller [20], Optimal Controller [21], Robust Controller [22], Sliding Mode Controller [23], Predictive Controller [24], and others. Although, these are well-accepted control methods for LCL currents control, a critical drawback of them is their limited range of operation, once these controllers have fixed gains. This issue turns relevant when the grid changes its conditions, from strong to weak and vice-versa, due to grid impedance variation [25]. This variation affects directly the resonance peak frequency of LCL filter, which consequently occurs at uncertain frequencies and can turn the control loop unstable. Among the fixed gains controllers, robust controllers are most successful methods to deal with grid-connected power systems, because they are designed for an operation range. However, to ensure a good performance, it requires a very accurate mathematical model of the plant and its control design can be grounded on complex mathematical theories, such as LMI (Linear Matrix Inequalities)-based controllers (see [26]). As alternative, adaptive controllers have been proposed to deal with large grid impedance variations and variable operation points. Some interesting works were shown on [27]-[30].

In [28], a discrete-time direct MRAC (Model Reference Adaptive Control) was proposed to control the converter- 
side currents of a static grid-connected converter with LCL. In this work, authors designed a second order reference model, using a Least Square-based algorithm for parametric adaptation. This control approach shown that grid-side currents were properly injected on the grid, supported by experimental results. By the other hand, in [27], [29], the control of grid-injected currents were made over grid-side currents. In these works, direct RMRAC (Robust MRAC) methods were proposed, also with adaption law based on Least Squares algorithms. In these works, third order reference models were designed. The presented results shown that controllers are robust to the grid uncertainties and also reject exogenous disturbances without need conventional PR Controllers. However, both controllers require high processing capacity, once the parametric adaptation law is based on Least Squares algorithms, which has a relevant amount of matrices multiplications. By the other hand, in [30], a discrete-time state-feedback RMRAC was proposed. In this approach, the adaptive identifier was a gradient-type algorithm, which implies significantly less computational burden. Although the presented results indicate robustness and fast current tracking, it also depends on a third order reference model. Due to the use of a high order reference model on control design, the control law still have to calculate too many gains online. Furthermore, as the controllers were designed to the gridinjected currents control, in $\alpha \beta$ coordinates, then there are twice the number of gains to calculate them online. This high quantity of operations can turn the controller infeasible in a restrict memory microcontroller, besides make the design and implementation a hard task. In addition, as the control is based on state-feedback, it requires a large set of sensors, to avoid implement state observers.

In addition to adaptive controllers based on the MRAC theory, there are a large amount of controllers that are developed using intelligent or iterative techniques. In this bias, some interesting works are: adaptive PI based on Fuzzy techniques [31], Neural Network PI Controller [32], robust controller optimised by genetic algorithms [33], [34] and so on. However, these controllers have a heavy and complex mathematical background, to design and implement them.

Thereby, in alternative to the discussed studies, in this work is presented a novel direct discrete-time robust adaptive PI controller. The controller is developed using RMRAC theory, completely on discrete-time. However, an unitary reference model is considered on controller design. In practice, it means that controller tracks straightforwardly the reference currents and there no need to implement the reference model. With it, a great portion of operations required by RMRAC methods are avoided, simplifying significantly the controller design. Mathematically, this approach results in a reformulation of parameters vector used for adaptation of adjustable gains. It turns the controller robust to unmodelled dynamics, while avoid the complexity inherent to the conventional high order robust model reference adaptive controllers, consequently implying in a lower computational burden. The development is presented on the text in a light way, with focus on implementation concepts and the mathematical proofs (robustness analysis, signal limitation and controller stability proof) are available on Appendix A.
Moreover, the controller is based only on outputfeedback, requiring a reduced set of sensors, for grid-side currents. Furthermore, the controller does not need multiloop controllers for grid disturbance rejection, or require any knowledge of grid parameters, lines impedance or load power demand. Also, due to its robust adaptive nature, it allows a considerable range of values for design parameters, as long as it respects the controller's restrictions, provided from robustness analysis. This controller is applied on grid-side currents control of a static 3-wire grid-connected VSC with LCL filter. A comparison of proposed controller and a robust adaptive controller is provided, assessing their performance and computational burden experimentally.

The regarding of this work is given as follows: on Section II, the LCL model is shown. Next, on Section III, the mathematical development of robust adaptive PI controller is presented. In Section IV, the experimental results are shown, besides a performance and computational burden comparison with an RMRAC. Finally, on Section V, the conclusions of this work are given.

\section{MATHEMATICAL MODEL OF LCL FILTER}

The modelling of static 3-wire grid-connected VSC with LCL filters is well discussed on [27]-[29]. Then, here, the model will be briefly presented. In the Figure 1, the electrical diagram of complete system is presented, including primary energy source, converter, LCL filter, the grid, microcontroller DSP (Digital Signal Processor) and sensors. Firstly, to turn easier the modelling, the converter switches are considered ideal and the grid is assumed to be predominantly inductive. The grid, $v_{d}$, is modelled as a sinusoidal source, in series with an inductance $L_{g 2}$ and parasitic resistance $R_{g 2}$. It is highlighted that LCL circuit is represented by the Thevenin equivalent in relation to the PCC (Point of Common Coupling) [29].

The LCL filter is compounded by converter-side inductors $L_{c}$, with associated parasitic resistance $R_{c}$, capacitors $C$ and grid-side inductors $L_{g 1}$, with associated parasitic resistance $R_{g 1}$. The total grid-side inductance $L_{g}$ and total grid-side resistance $R_{g}$ are given by $L_{g}=L_{g 1}+L_{g 2}$ and $R_{g}=R_{g 1}+R_{g 2}$, respectively. In Figure 1, the sensors are also shown as inputs on DSP. The sensors are used to measure grid-side currents $\left(i_{g a}, i_{g b}\right.$ and $\left.i_{g c}\right)$ and voltage at PCC $\left(v_{a b}\right.$ and $\left.v_{b c}\right)$. In addition, $V_{c c}$ is the DC link voltage, which represents the primary energy source that can be a solar panel, wind turbine or other clean energy source. Moreover, DSP stores the measures, computes the control law and synthesises it through SVM (Space Vector Modulation) technique.

It is complex to design a controller using three-phase LCL model in $a b c$ coordinates, once their parameters are strongly coupled, as discussed on [28], [29]. Then, the model is converted to two identical decoupled single-phase linear invariant-time systems, in $\alpha \beta 0$ coordinates, through Clarke Transform [35]. The electrical diagram of two decoupled systems, considering equilibrated phases, is shown on Figure 2 , where $\alpha$ and $\beta$ are the indexes for $\alpha$ and $\beta$ coordinates, respectively. Note that considering equilibrated phases, there is no path for current conduction on the $\mathbf{0}$ axis, then, it can be disregarded.

Considering converter disconnected from the grid, the 


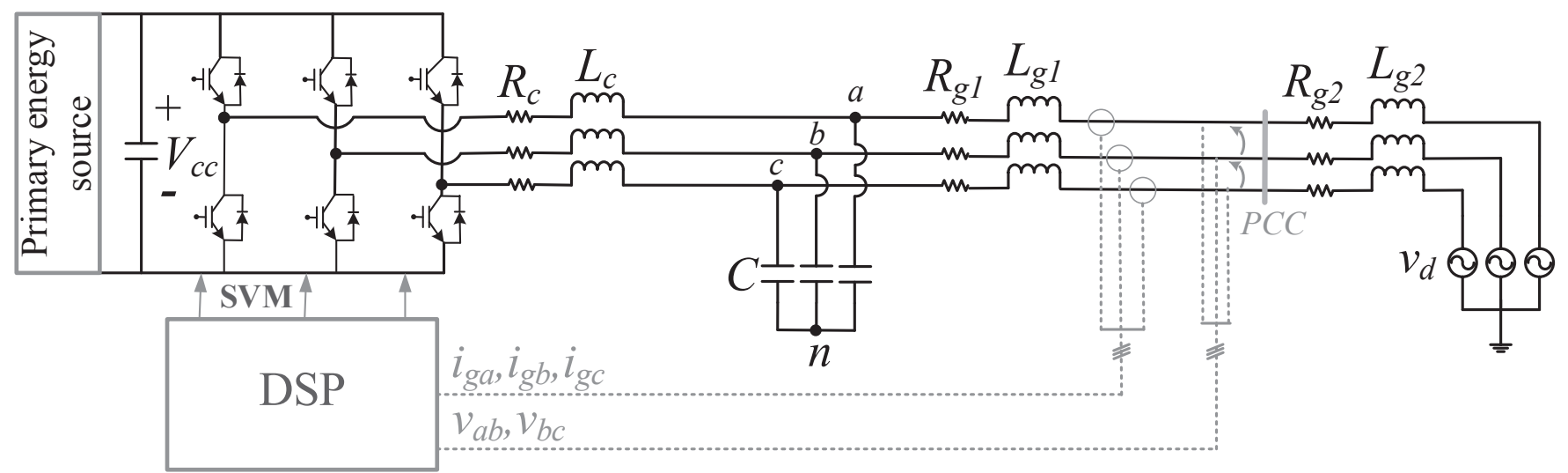

Fig. 1. Electrical diagram of static 3-wire grid-connected VSC with LCL filter
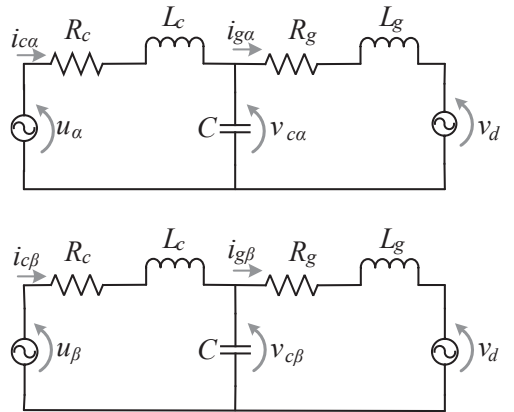

Fig. 2. Electrical diagram of equivalent circuit of static 3-wire gridconnected VSC with LCL filter, considering equilibrated phases

identical transfer function of decoupled single-phase systems, for $\alpha$ and $\beta$ coordinates, is given by

$$
\frac{i_{g}(s)}{u(s)}=\frac{\frac{1}{L_{g} L_{c} C}}{s^{3}+\frac{\left(R_{g} L_{c}+R_{c} L_{g}\right)}{L_{g} L_{c}} s^{2}+\frac{\left(L_{c}+L_{g}+R_{g} R_{C} C\right)}{L_{g} L_{c} C} s+\frac{R_{g}+R_{c}}{L_{g} L_{c} C}},
$$

where $u(s)$ is the voltage synthesised by converter through modulation technique.

\section{DISCRETE-TIME ROBUST ADAPTIVE PI CONTROLLER}

The novel robust adaptive controller is developed using PI and RMRAC theories. Then, initially, consider the plant described as follows,

$$
y=G(z) u,
$$

where $G(z)$ and $y$ are the complete plant transfer function and its output, respectively. The $G(z)$ can be subject to unmodelled dynamics, as described by

$$
G(z)=G_{0}(z)\left[1+\mu_{m} \Delta_{m}(z)\right]+\mu_{a} \Delta_{a}(z),
$$

where $\mu_{a} \Delta_{a}(z)$ and $\mu_{m} \Delta_{m}(z)$ are additive and multiplicative dynamics, respectively. With no loss of generality, the constants $\mu_{a}$ and $\mu_{m}$ can be identical $\left(\mu_{a}=\mu_{m}=\mu\right)$ [36], as will be used on robustness analysis, discussed on Appendix A.
Furthermore, $G_{0}(z)$ is the nominal part of the plant, given by

$$
G_{0}(z)=k_{p} \frac{Z_{0}(z)}{R_{0}(z)}
$$

where $k_{p}$ is the high frequency gain of the plant, $Z_{0}(z)$ and $R_{0}(z)$ are monic polynomials with degree $m$ and $n$, respectively.

The nominal part of the plant, $G_{0}(z)$, have to satisfy the following assumptions [36],

$A 1$ ) The signal of $k_{p}$ and degrees $m$ and $n$ are known;

A2) $Z_{0}(z)$ is Schur polynomial with degree $m \leq n-1$;

A3) $\Delta_{m}(z)$ is a stable transfer function and $\Delta_{a}(z)$ is a strictly proper transfer function;

A4) An upper bound, $p_{0}$, is known and the poles of $\Delta_{a}(z-p)$ and $\Delta_{m}(z-p)$ are stable, internal to the unit radius circle $|z|$, $0<p<1$.

The mathematical development of the controller starts from discrete-time PI controller equation [37], which is given by

$$
u(z)=\left(K_{p}+\frac{K_{i}}{1-z^{-1}}\right) e_{0}(z)
$$

and in its implementable form can be written as

$$
u(k)=u(k-1)+\left(K_{p}+K_{i}\right) e_{0}(k)-K_{p} e_{0}(k-1),
$$

where $K_{p}$ and $K_{i}$ are proportional and integral gains, respectively. Moreover, $e_{0}(k)$ is the regulation error, given by

$$
e_{0}(k)=r(k)-y(k)
$$

where $r(k)$ is the limited reference signal.

To improve adaptability to the PI controller, the RMRAC theory is used to allow adjust gains online. To achieve it, firstly, the regulation error is replaced in (6) and dividing the result by $-\left(K_{p}+K_{i}\right)$, which follows

$$
\begin{array}{r}
\frac{-1}{K_{p}+K_{i}} u(k)+\frac{1}{K_{p}+K_{i}} u(k-1)-y(k)-\ldots \\
\cdots \frac{K_{p}}{K_{p}+K_{i}} e_{0}(k-1)+r(k)=0 .
\end{array}
$$


From (8), follows the new PI control law for adaptation, turning each term of control law in an adjustable gain, differently from works previously discussed that adapts specifically the $K_{p}$ and $K_{I}$ gains or PI control action. It results in

$$
\begin{array}{r}
\theta_{1}(k) u(k)+\theta_{2}(k) u(k-1)+\theta_{3}(k) y(k)+\ldots \\
\ldots \theta_{4}(k) e_{0}(k-1)+r(k)=0 .
\end{array}
$$

Note that (9) is a reformulation of parameters vector used for adaptation of adjustable gains, and, as outcome, a new controller is developed. However, (9) does not take into account exogenous disturbances rejection, which is necessary for power systems connected to the grid. To achieve it, consider that disturbances are described as

$$
\begin{gathered}
V_{s}(k)=A_{s} \sin \left(\omega_{d_{s}} k T_{s}+\phi_{s}\right), \\
V_{c}(k)=A_{c} \cos \left(\omega_{d_{c}} k T_{s}+\phi_{c}\right),
\end{gathered}
$$

where $V_{s}(k)$ and $V_{c}(k)$ are the components of phase and quadrature of the exogenous disturbances, respectively. In addition, $A, \phi$ and $\omega_{d}$ are the amplitude, phase and frequency of the components on phase and quadrature of exogenous disturbances, respectively.

Then, considering the terms of disturbance rejection in (9), it follows,

$$
\begin{array}{r}
\theta_{1}(k) u(k)+\theta_{2}(k) u(k-1)+\theta_{3}(k) y(k)+\theta_{4}(k) e_{0}(k-1)+\ldots \\
\ldots \theta_{s}(k) V_{s}(k)+\theta_{c}(k) V_{c}(k)+r(k)=0,
\end{array}
$$

or, in vector representation,

$$
\boldsymbol{\theta}^{T}(k) \boldsymbol{\omega}(k)+r(k)=0,
$$

where the auxiliary vector $\boldsymbol{\omega}(k)$ is composed by

$$
\boldsymbol{\omega}^{T}(k)=\left[u(k) u(k-1) y(k) e_{0}(k-1) V_{s}(k) V_{c}(k)\right],
$$

and the adaptive gains vector is formed by

$$
\boldsymbol{\theta}^{T}(k)=\left[\theta_{1}(k) \theta_{2}(k) \theta_{3}(k) \theta_{4}(k) \theta_{s}(k) \theta_{c}(k)\right] .
$$

Note that there is no reference model, $W_{m}(z)$, to design, as is commonly seen on RMRAC projects. In this work, it was considered as an ideal model, that is, $W_{m}(z)=$ 1. It was assumed with the aim to reduce controller complexity. Thereby, due to it, the reference currents are tracked straightforwardly, once $y_{m}=W_{m}(z) r=r$, where $y_{m}$ is the model reference output. Therefore, the controller objective is defined as: given a limited reference signal $r(k)$, for some $\mu^{*}>0$ and any $\mu \in\left[0, \mu^{*}\right)$, the closed-loop controller will be globally stable and the plant output will track the reference signal as closest as possible, even if the plant is subject to unmodelled dynamics, $\Delta_{m}(z)$ and $\Delta_{a}(z)$. As consequence, the computational burden to execute it experimentally is reduced, because a great part of operations required by RMRAC methods are avoided.

In order to adapt the parameters, a Gradient-modified algorithm is used, which is given as follows,

$$
\boldsymbol{\theta}(k+1)=\boldsymbol{\theta}(k)-T_{s} \boldsymbol{\sigma}(k) \boldsymbol{\Gamma} \boldsymbol{\theta}(k)-T_{s} \kappa \boldsymbol{\Gamma} \frac{\boldsymbol{\omega}(k) e_{0}(k)}{\bar{m}^{2}(k)},
$$

where $\Gamma$ is a positive defined symmetric matrix, which defines the convergence rate, as well as, the positive scalar $\kappa$, used to accelerate gain adaptation. Also, $T_{S}$ is the sampling time.

The majorant signal $\bar{m}(k)$ is used to improve robustness to the parametric adaptation algorithm. This signal is similar to a normaliser, and it is calculated as follows,

$$
\bar{m}^{2}(k)=m^{2}(k)+\boldsymbol{\omega}^{T}(k) \boldsymbol{\Gamma} \boldsymbol{\omega}(k),
$$

where,

$$
m(k+1)=\left(1-T_{s} \delta_{0}\right) m(k)+T_{s} \delta_{1}(1+|u(k)|+|y(k)|),
$$

and the initial condition have to satisfy $m(0) \geq \delta_{1} /\left(1-\delta_{0}\right)$ [36]. Furthermore, the $\sigma$-modification [38], which also contributes to the robustness of the parametric adaptation algorithm, avoiding parameters drifting, is given by

$$
\sigma(k)=\left\{\begin{array}{lll}
0 & \text { if } & \|\boldsymbol{\theta}(k)\| \leq M_{0} \\
\sigma_{0}\left(\frac{\|\boldsymbol{\theta}(k)\|}{M_{0}}-1\right) & \text { if } & M_{0}<\|\boldsymbol{\theta}(k)\|<2 M_{0}, \\
\sigma_{0} & \text { if } & \|\boldsymbol{\theta}(k)\| \geq 2 M_{0}
\end{array}\right.
$$

where $M_{0}>\left\|\boldsymbol{\theta}^{*}\right\|$ in an upper bound of $\boldsymbol{\theta}^{*}$ norm, oversized due to no knowledge of $\left\|\boldsymbol{\theta}^{*}\right\|$ and $\sigma_{0}$ is the upper bound of $\sigma$-modification.

A block diagram of this controller is shown on Figure 3. Note that regulation error is given directly between reference signal and plant output, to be used on gradient-modified algorithm, where the gains are calculated, limited by majorant signal and $\sigma$-modification. The robustness analysis, signal limitation and controller stability are discussed on Appendix A.

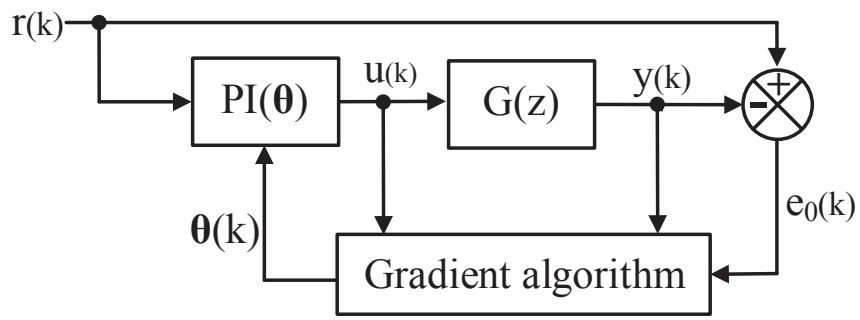

Fig. 3. Block diagram of discrete-time Robust Adaptive PI controller

The steps to implement the presented controller are:

1. Update of reference $r(k)$

2. Update of auxiliary signal $\boldsymbol{\omega}(k)$

3. Update of gains norm $\|\boldsymbol{\theta}(k)\|$

4. Update of $\sigma$-modification $\sigma(k)$

5. Update of regulation error $e_{0}(k)$

6. Update of majorant signal $\bar{m}^{2}(k)$

7. Update of gains $\boldsymbol{\theta}(k+1)$

8. Update of control action $u(k)$ 


\section{EXPERIMENTAL RESULTS}

To evaluate the performance of the presented controller, it was compared with other robust adaptive controller, described on next subsection.

The controllers were implemented in a laboratory prototype, using a DSP TMS320F28335 Delfino microcontroller from Texas Instruments. The prototype has the characteristics $\left(L_{c}, C\right.$ and $\left.L_{g 1}\right)$ from Table I, whereas the parameters $R_{g 2}$ and $L_{g 2}$ are unknown. It is highlighted that filter was designed following the steps presented on [39] that guarantee a good attenuation of harmonics on grid side and low reactive power consumption by capacitors. The experimental setup is show on Figure 4.

\section{TABLE I}

\section{LCL Filter Parameters}

\begin{tabular}{ccc}
\hline Symbol & Parameter & Value \\
\hline$L_{c}$ & Converter-side inductance & $1 \mathrm{mH}$ \\
\hline$R_{C}$ & Converter-side resistance & $50 \mathrm{~m} \Omega$ \\
\hline$C$ & Capacitance of LCL filter & $62 \mu \mathrm{F}$ \\
\hline$L_{g 1_{\min }}$ & Grid-side inductance & $0.3 \mathrm{mH}$ \\
\hline$L_{g 1_{\max }}$ & Grid-side inductance & $1.3 \mathrm{mH}$ \\
\hline$R_{g 1}$ & Grid-side resistance & $50 \mathrm{~m} \Omega$ \\
\hline
\end{tabular}

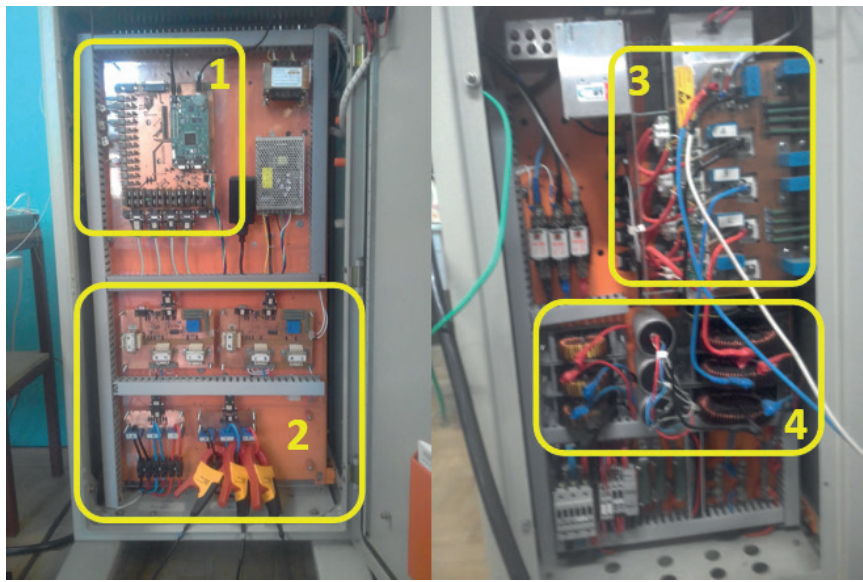

Fig. 4. Prototype: 1: DSP, 2: current sensors, 3: Converter and voltage sensors, 4: LCL filter

The line voltage range was set to $110 \mathrm{~V}$, adjusted by a threephase transformer. Moreover, the DC bus voltage was set to $500 \mathrm{~V}$. It is highlighted that this voltage is not controlled, however, it did not interfered in the controller performance. The converter's power is $5.4 \mathrm{~kW}$, its switching frequency and the controller's sampling frequency were both $5040 \mathrm{~Hz}$. By the other hand, the controller's data (adaptive gains and regulation errors) were saved one sample every four controller interruptions. It was necessary to store sufficient data to evaluate the convergence of adaptive controller parameters, once there was not used external memory on DSP.

Furthermore, the test starts with $L_{g 1_{\min }}=0.3 \mathrm{mH}$ and to evaluate the controller performance, an additional inductance with $1 \mathrm{mH}$ is added at a certain instant of the test. With it, the LCL grid-side inductance changes to $L_{g 1_{\max }}=1.3 \mathrm{mH}$. However, the total grid-side inductance is unknown, once grid inductance is uncertain.

Furthermore, a Kalman Filter [40] is used to synchronise the controller with the grid voltages, measured on PCC. In addition, from this synchronisation, the phase voltages of the PCC, in $\alpha \beta$ coordinates, are used to obtain the phase and quadrature signals of grid fundamental component, used for disturbances rejection and also used for current reference generation [28].

The total time of the experiment is around 1.6s. The initial current reference amplitude was set to $20 \mathrm{~A}$ and it is increased to $30 \mathrm{~A}$ after $0.4 \mathrm{~s}$. Besides, at a certain instant of the experiment $(0.4 s$ after reference be changed), a parametric variation is imposed on the grid impedance, by adding a $1 \mathrm{mH}$ in series with the grid, as previously discussed. This routine test was used for robust adaptive PI controller and the high order RMRAC, to compare their performance and computational burden, experimentally. These results can be seen on the next two subsections.

\section{A. Robust Model Reference Adaptive Controller}

The controller implemented to realise the comparison of performance and computational burden is the RMRAC, shown on [41], which is based on [42]. This control technique tracks the output $y_{m}$ of a reference model $W_{m}(z)$, which has the same relative degree that nominal part of the plant $G_{0}(z)$. For LCL filter control, to implement this RMRAC, it is necessary a third order reference model, which is not required by proposed robust adaptive PI controller, once it tracks straightforwardly the reference currents.

Then, for RMRAC, the error is given by $e_{1}(k)=y(k)-$ $y_{m}(k)$ and the control action, $u$, is implemented from

$$
\boldsymbol{\theta}^{T}(k) \boldsymbol{\omega}(k)+r(k)=0,
$$

where $\boldsymbol{\theta}(k)$ is the adaptive gains vector and $\boldsymbol{\omega}(k)$ is a parametric vector. The $\boldsymbol{\omega}(k)$ vector is composed by reconstructive filters $\boldsymbol{\omega}_{1}(k)$ and $\boldsymbol{\omega}_{2}(k)$, plant output and control action. The reconstructive filters are given by

$$
\begin{aligned}
& \boldsymbol{\omega}_{1}(k+1)=\left(\boldsymbol{I}+\boldsymbol{F} T_{s}\right) \boldsymbol{\omega}_{1}(k)+\boldsymbol{q} T_{s} u(k), \\
& \boldsymbol{\omega}_{2}(k+1)=\left(\boldsymbol{I}+\boldsymbol{F} T_{s}\right) \boldsymbol{\omega}_{2}(k)+\boldsymbol{q} T_{s} y(k),
\end{aligned}
$$

where $\boldsymbol{I}$ is an identity matrix of dimensions $n \times n$ and $(\boldsymbol{F}, \boldsymbol{q})$ is a controllable pair with a stable matrix $\boldsymbol{F}$ and a controllable parameters vector $\boldsymbol{q}$, with dimension $n-1 \times n-1$ and $n-1$, respectively [38].

Thus, the gradient algorithm changes to

$$
\boldsymbol{\theta}(k+1)=\boldsymbol{\theta}(k)-T_{s} \boldsymbol{\sigma}(k) \boldsymbol{\Gamma} \boldsymbol{\theta}(k)-T_{s} \kappa \frac{\boldsymbol{\Gamma} \boldsymbol{\zeta}(k) \boldsymbol{\varepsilon}(k)}{m^{2}(k)},
$$

where the augmented error, $\varepsilon(k)$, is

$$
\varepsilon(k)=e_{1}(k)+\boldsymbol{\theta}^{T}(k) \boldsymbol{\zeta}(k)-y_{m}(k),
$$

and the auxiliary vector $\zeta$ is

$$
\zeta=W_{m}(z) \boldsymbol{\omega}
$$

being $\boldsymbol{\zeta}(k)$ the vector $\boldsymbol{\omega}(k)$ filtered by $W_{m}(z)$. For LCL filter control, which is third order system, $\boldsymbol{\omega}(k)$ is composed by $\boldsymbol{\omega}(k)=\left[\begin{array}{llllll}\omega_{11}(k) & \omega_{12}(k) & \omega_{21}(k) & \omega_{22}(k) & y(k) & u(k)\end{array}\right.$ $\left.V_{s}(k) V_{c}(k)\right]^{T}$. Consequently, $\boldsymbol{\theta}(k)=\left[\begin{array}{ll}\theta_{11}(k) & \theta_{12}(k)\end{array}\right.$ 
$\left.\theta_{21}(k) \theta_{22}(k) \theta_{y}(k) \theta_{u}(k) \theta_{s}(k) \theta_{c}(k)\right]^{T}$. It is highlighted that $\sigma$-modification is the same as (18), as well the majorant signal, described on (16)-(17).

The design parameters of RMRAC are $\Gamma=40 I, \kappa=1000$, $\sigma_{0}=0.1, M_{0}=10, \delta_{0}=0.7$ and $\delta_{1}=1$, as defined on [41], as well as the reference model,

$$
W_{m}(z)=\frac{0.343}{(z-0.3)^{3}}
$$

and the initial gains,

$$
\boldsymbol{\theta}_{\alpha}(0)=\left[\begin{array}{c}
-2.3075082 \\
0 \\
-0.65603852 \\
0 \\
-1.0379406 \\
-1.9491602 \\
3.3076313 \\
-0.36709696
\end{array}\right] \quad, \quad \boldsymbol{\theta}_{\beta}(0)=\left[\begin{array}{c}
-0.84257501 \\
0 \\
-0.32428530 \\
0 \\
-0.83423382 \\
-1.2983845 \\
1.5830313 \\
-0.11256287
\end{array}\right] .
$$

It is highlighted that gains were set by choosing final gains of a simulation, used to be initialised closer to $\boldsymbol{\theta}^{*}$ and avoid excessive overshoot in the initial transient. However, they can be initialised with any value, which will converge. The only restriction is the signal of $\theta_{u}$, which have to be correct to avoid division by zero on implementation of (19).

The initial transient regime is shown on Figure5. Note that, even with gains initialisation, a short transient regime is observed due to grid uncertainties. This transient takes around $16 \mathrm{~ms}$ and no excessive overshoot is observed.

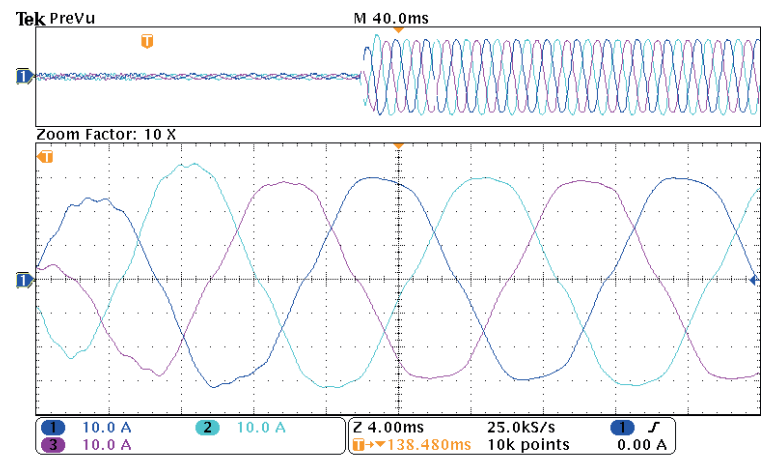

Fig. 5. Initial transient regime of grid-injected currents

In the Figure 6 is shown the reference amplitude change transient, from $20 \mathrm{~A}$ to $30 \mathrm{~A}$. It takes around $12 \mathrm{~ms}$ to achieve the new current references, faster than initial transient. Also, no overshoot occurred while controller gains are readapting.

As was aforementioned, to evaluate controller performance, an additional inductance in series with the grid is triggered at a certain instant. This instant can be seen on Figure 7. Note that the controller acts fast to compensate the impedance variation and only a small distortion is caused by grid condition change. These distortions, observed on currents tracking, is corrected in around $12 \mathrm{~ms}$. Furthermore, no excessive overshoot is observed.

Finally, the steady state regime is shown on Figure 8. As can be seen, the controller maintain the grid-injected currents with proper amplitude, which is $30 \mathrm{~A}$. The THD in steady state

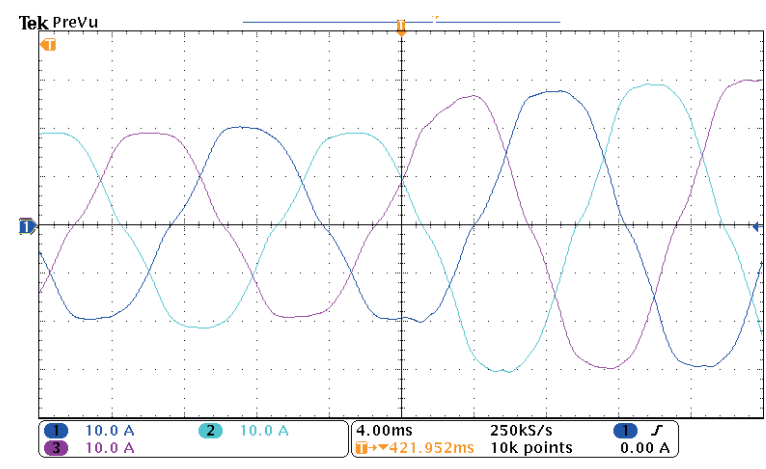

Fig. 6. Grid-injected currents in the reference change transient

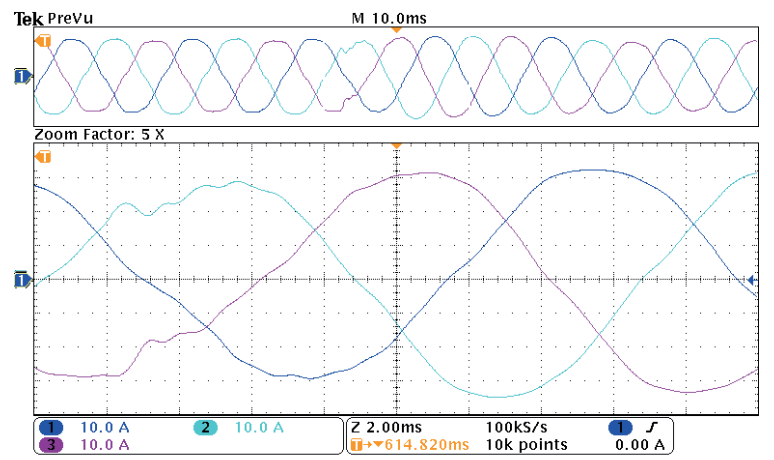

Fig. 7. Grid-injected currents in the parametric variation transient

regime is $2.48 \%$, respecting the IEEE 1547 .

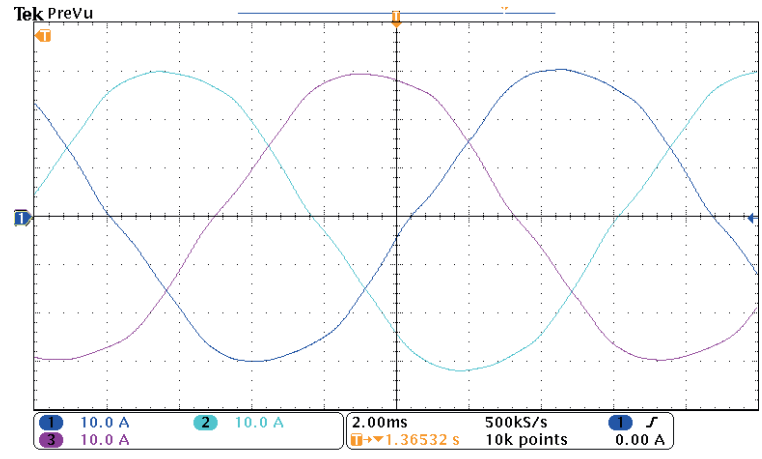

Fig. 8. Grid-injected currents in steady state

As the controller was designed on $\alpha \beta$ coordinates, the tracking errors in these coordinates are shown on Figure 9, complementing the currents information from oscilloscope, previously presented. As can be seen, the tracking errors remain small and converge to a residual set asymptotically.

To evaluate the controller parameters convergence, the $\boldsymbol{\theta}(k)$ gains, in $\alpha$ and $\beta$ coordinates, are presented in Figure 10 and Figure 11, respectively. Note that gains converge fast, and, as they were properly initialised, there are small transient regime. Besides, when references change the gains readapt fast. The same behaviour can be observed when the grid condition was altered, once the gains are closer to an ideal set. By consequence, the transient regimes for reference change and when parametric variation occurred were shorter than initial transient.

As expected, the RMRAC has a good performance when applied to grid-side currents control of a VSC with LCL 


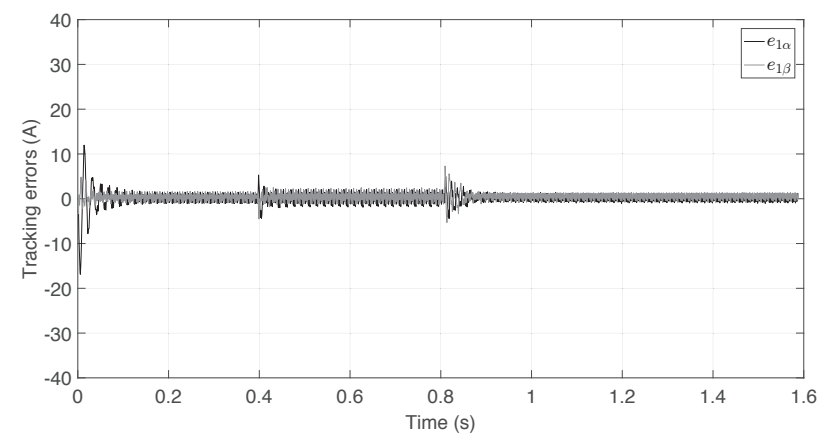

Fig. 9. Tracking errors in $\alpha \beta$ coordinates

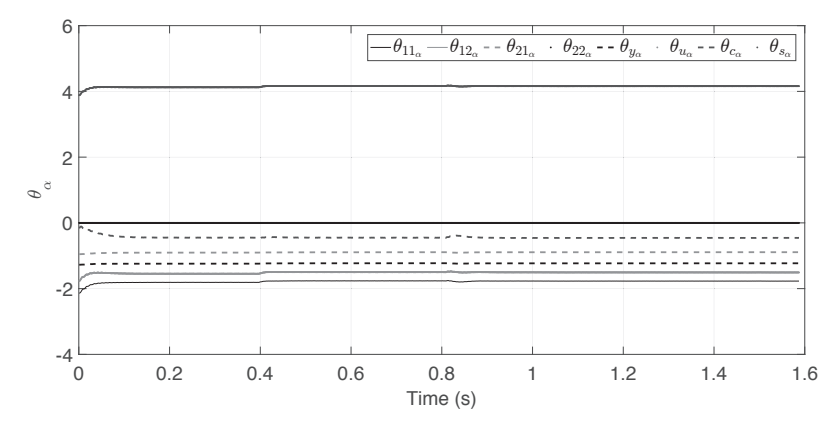

Fig. 10. Gains in $\alpha$ coordinate

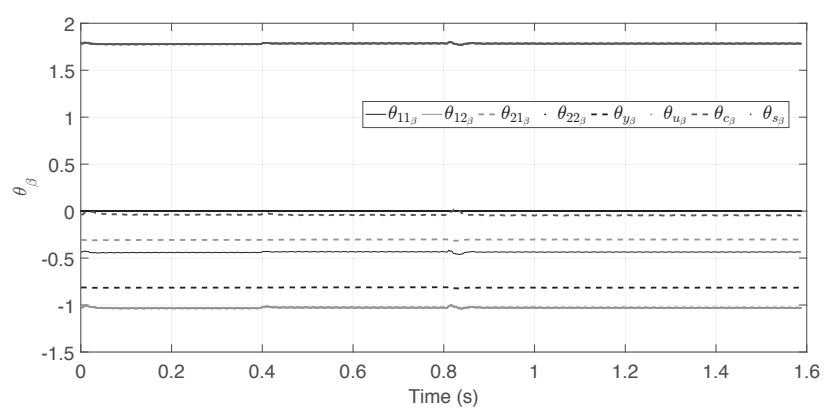

Fig. 11. Gains in $\beta$ coordinate

filter. However, to achieve this high performance, it requires a large set of operations (182 addition/subtraction and 446 multiplication/division), which requires $37.56 \mu$ s to execute it.

\section{B. Robust Adaptive PI Controller}

Due to robust adaptive nature of the controller, it allows a considerable range of values for design parameters, as long as it respects the controller's restrictions, provided from robustness analysis, presented on Appendix A. However, for fast practical implementation, the parameters can be set as follows: $\Gamma \kappa T_{s} \leq 20 ; \delta_{0}$ and $\delta_{1}$ are chosen to act as a slow dynamics filter to smooth $\bar{m}(k)$ response; and $M_{0}$ can be securely oversized, $M_{0} \geq 2\left\|\boldsymbol{\theta}^{*}\right\|$, as discussed on [42]. The design parameters are $\Gamma=500, \kappa=1000, \sigma_{0}=0.1, M_{0}=15$, $m^{2}(0)=4, \delta_{0}=0.7$ and $\delta_{1}=1$.

To initialise the gains $\boldsymbol{\theta}(0)$, in $\alpha$ and $\beta$, a simulation was performed and the final values of $\boldsymbol{\theta}(k)$ were used as initial values of the experiment. It is emphasised that it is only used to avoid excessive overshoot in the initial transient regime, once high currents can damage the prototype. If the application does not have security issues, the gains can be initialised with random values. For this application, the initial gains were set as

$$
\boldsymbol{\theta}_{\alpha}(0)=\left[\begin{array}{c}
1.4666969 \\
1.4666969 \\
-1.0000000 \\
-8.3924341 \\
-2.9755771 \\
-0.4001412
\end{array}\right] \quad, \quad \boldsymbol{\theta}_{\beta}(0)=\left[\begin{array}{c}
1.4994920 \\
1.4994920 \\
-1.0000000 \\
-8.3349009 \\
-2.8854203 \\
-0.0643255
\end{array}\right] .
$$

The initial transient regime is shown on Figure12. Note that, even while parameters are converging for a set of limited gains close to $\boldsymbol{\theta}^{*}$, it presents no excessive overshoot, thanks to proper gains initialisation. Due to the grid uncertainties, a short transient regime is observed. It takes around $20 \mathrm{~ms}$, to adjust their gains and regulate the grid-injected currents with desired amplitude reference, which is $20 \mathrm{~A}$.

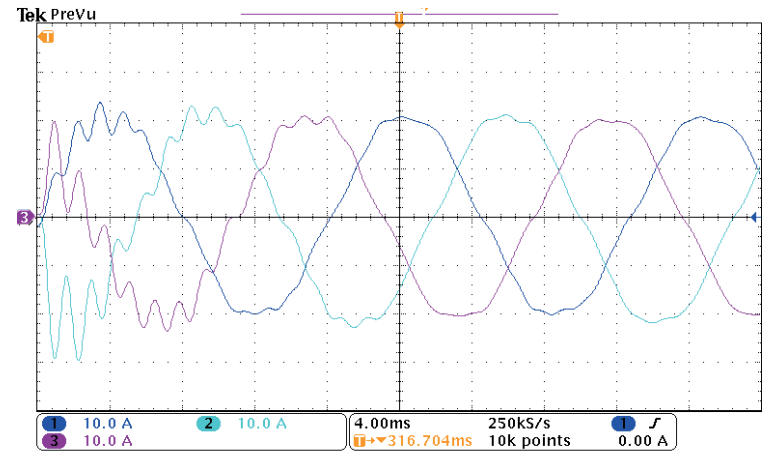

Fig. 12. Initial transient regime of grid-injected currents

In the Figure 13 is shown the instant that reference amplitude is changed from $20 \mathrm{~A}$ to $30 \mathrm{~A}$. Again, the currents tracks fast the references. A small distortion is observed on measured currents, which occurs while the controller is readapting their gains for the new nominal point of operation. Note that it takes around $12 \mathrm{~ms}$, less time than initial transient, once the gains in this situation are close than an ideal set of gains. Moreover, although some oscillations occur in this transient, no relevant overshoot affects the currents while controller adjust their gains.

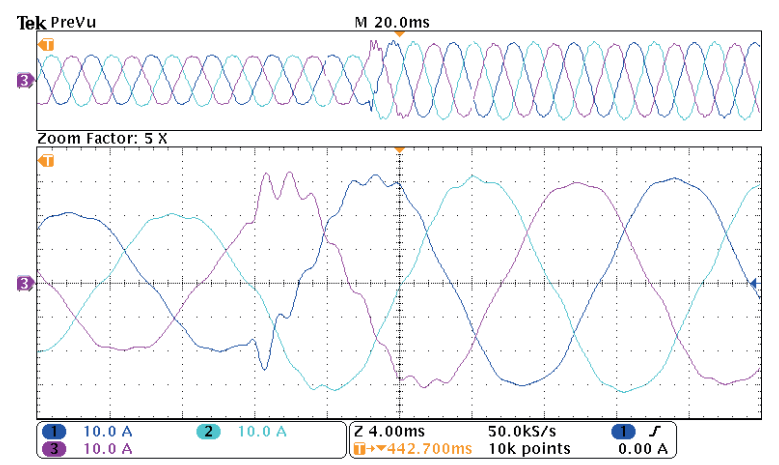

Fig. 13. Grid-injected currents in the reference change transient

As was aforementioned, to evaluate controller performance, an additional inductance in series with the grid is triggered at a certain instant. This instant can be seen on Figure 
14. Note that the controller acts fast to compensate the impedance variation and only a small distortion is caused by grid condition change. These distortions, observed on currents tracking, is corrected in around $6 \mathrm{~ms}$.

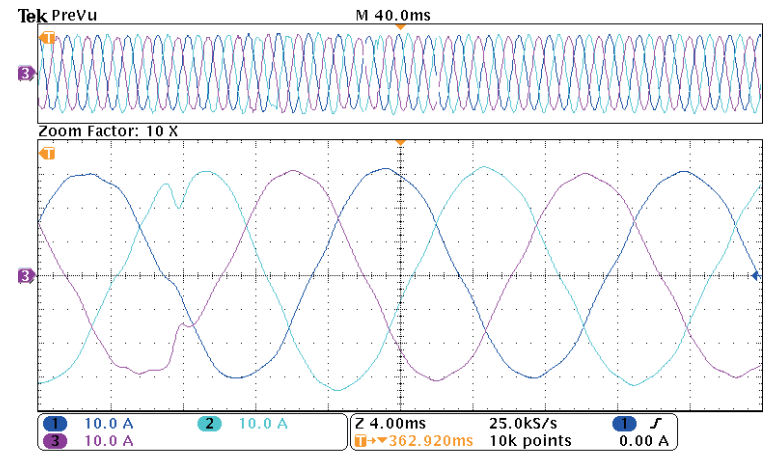

Fig. 14. Grid-injected currents in the parametric variation transient

Finally, the steady state regime is shown on Figure 15. As can be seen, the controller maintain the grid-injected currents with proper amplitude, which is $30 \mathrm{~A}$. The THD in steady state regime is $2.47 \%$, respecting the IEEE 1547 and similar to the high order RMRAC.

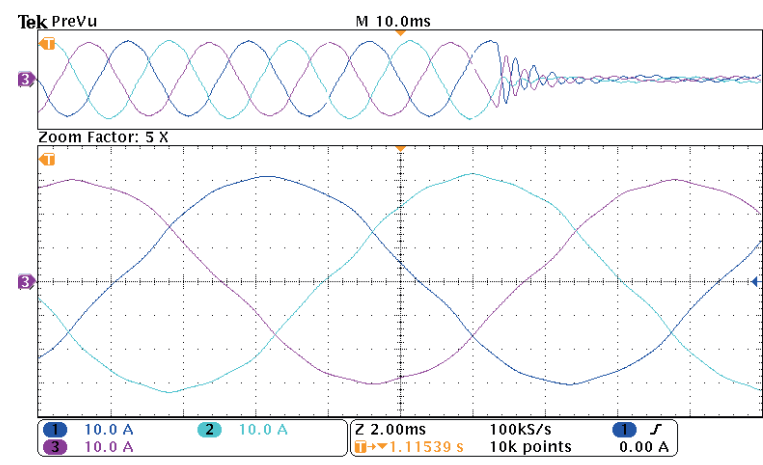

Fig. 15. Grid-injected currents in steady state

As the controller was designed on $\alpha \beta$ coordinates, the regulation errors in these coordinates are shown on Figure 16, complementing the currents information from oscilloscope, previously presented. As can be seen, the regulation errors remain small and converge to a residual set asymptotically.

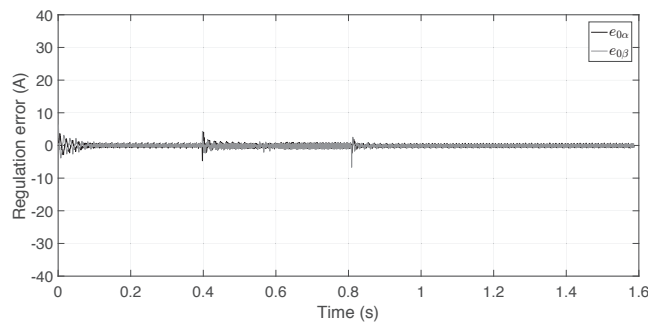

Fig. 16. Regulation errors in $\alpha \beta$ coordinates

To evaluate the controller parameters convergence, the $\boldsymbol{\theta}(k)$ gains, in $\alpha$ and $\beta$ coordinates, are presented in Figures 17 and 18 , respectively. Note that gains converge fast, and, as they were initialised closer to ideal gains, there are small transient regime. Moreover, as the boundness of all closed-loop signals are ensured by majorant signal and the $\sigma$-modification being used to avoid parameters drift, no bursting phenomena (a kind of local instability) occurred during the experiment.

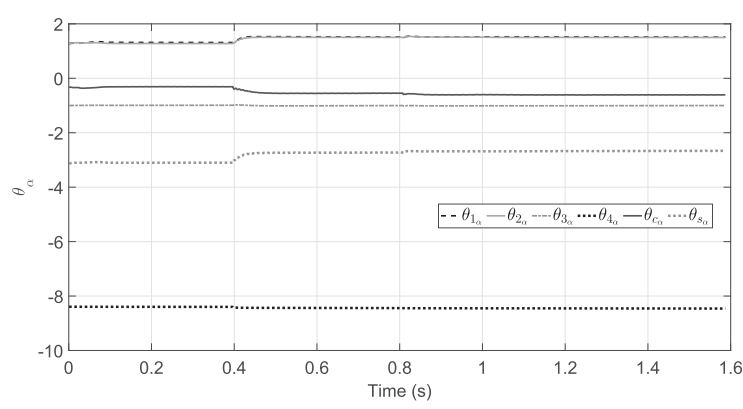

Fig. 17. Gains in $\alpha$ coordinate

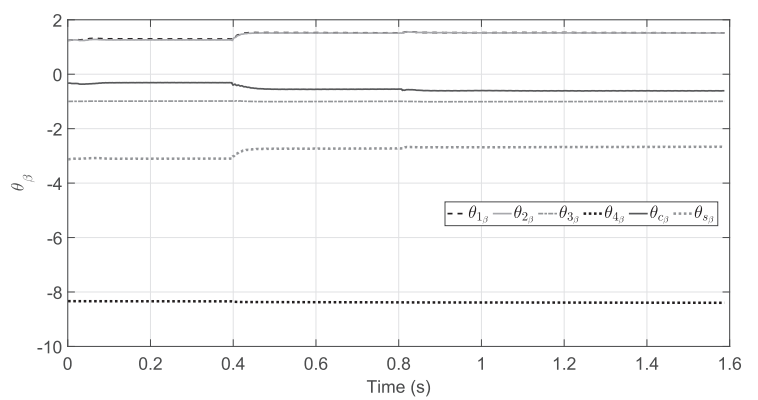

Fig. 18. Gains in $\beta$ coordinate

In comparison with RMRAC, the robust adaptive PI controller presented a similar performance, in regarding to current tracking error and THD, however, without a complex control design. It can be achieved by direct tracking of reference, which reduces computation delay and cumulative truncation error. Besides, the transient times of both were also similar. For robust adaptive PI controller, they are: $20 \mathrm{~ms}$, $12 \mathrm{~ms}$ and $6 \mathrm{~ms}$ for initial instant, reference change instant and parametric variation instant, respectively. For RMRAC, they are: $16 \mathrm{~ms}, 12 \mathrm{~ms}$ and $12 \mathrm{~ms}$, for initial instant, reference change instant and parametric variation instant, respectively. Then, in regarding to the performance, the controllers can be considered equivalent, once they presented similar transient time, THD and currents tracking errors.

By the other hand, in terms of computational burden, the robust adaptive PI controller requires a reduced set of operations, which implies in a relevant reduction of time processing. This set of operations consist in 102 addition/subtraction and 228 multiplication/division, which requires $21.96 \mu \mathrm{s}$ to execute it. Comparing with RMRAC, the robust adaptive PI controller reduces $41,64 \%$ of time processing, which alleviates significantly microcontroller requirements. Thereby, these results corroborate that presented controller is a feasible control strategy for grid-connected VSC with LCL filter, which maintain the performance of high order RMRAC and provides a simpler controller with reduced computational burden.

\section{CONCLUSION}

In this paper, a direct discrete-time robust adaptive PI controller was presented and used to control the grid-injected 
currents of a grid-connected VSC with LCL filter. By means of experimental results, it was verified the controller performance, which achieves fast convergence, equivalent to a high order RMRAC. The longer transient regime occurred on initial instants, when the gains are adapting more intensively, and takes around 20ms. After gains converged, the transient regime were shorter. They were $12 \mathrm{~ms}$ and $6 \mathrm{~ms}$ for reference change instant and parametric variation instant, respectively. Also, no relevant overshoot was observed in these transient regime. The advantages of proposed technique in relation to existing high order adaptive controllers is its reduced computational burden, simpler structure that tracks the references directly, intuitive parameters tuning, which has global stability ensured if designed respecting the robustness constraints, and small amount of sensors to implement it. In regarding to computational burden, the presented controller requires $41,64 \%$ less processing time than conventional high order RMRAC, maintaining a similar performance. Its THD in steady state regime was $2.47 \%$. Moreover, it presents another advantage in relation to other classical controller, such as the PI in synchronous frame, once the presented controller does not need to convert to $d q$ coordinates, and it does not require PR controllers to reject exogenous disturbances, commonly associated to fixed-gains controllers. Besides, the presented robust adaptive PI controller do not require any knowledge of grid parameters, lines impedance or load power demand to control the grid-side currents, and consequently the active or reactive power output of VSC, as well as others grid-connected power systems. Thus, the robust adaptive PI controller is a feasible controller, which does not require a high processing capacity.

\section{Appendix A - Robustness Analysis and Stability Proof}

Consider the plant described by (2)-(4), subject to assumptions $A 1$ to $A 4$, using the control law (12)-(14), and the adaptation algorithm (15)-(18), with limited reference, are able to find a parameters vector $\boldsymbol{\theta}(k)$ whose limitation of parametric error vector $\boldsymbol{\phi}(k)$ is ensured.

Following the steps shown in [36], it is possible to write the regulation error in function to unmodelled dynamics,

$$
e_{0}=-\left(\boldsymbol{\phi}^{T} \boldsymbol{\omega}+\mu \eta\right)
$$

where $\eta$ is unmodelled dynamics. Be a Lyapunov function candidate defined as

$$
V(k)=\boldsymbol{\phi}^{T}(k) \boldsymbol{\Gamma}^{-1} \boldsymbol{\phi}(k),
$$

where $\boldsymbol{\phi}(k)=\boldsymbol{\theta}(k)-\boldsymbol{\theta}^{*}$ and $\boldsymbol{\theta}^{*}$ are the ideal gains. Then, subtracting $\boldsymbol{\theta}^{*}$ in both sides of (15), then $\boldsymbol{\phi}(k)$ is obtained,

$$
\boldsymbol{\phi}(k+1)=\boldsymbol{\phi}(k)-T_{s} \boldsymbol{\sigma}(k) \boldsymbol{\Gamma} \boldsymbol{\theta}(k)-T_{s} \kappa \boldsymbol{\Gamma} \frac{\boldsymbol{\omega}(k) e_{0}(k)}{\bar{m}^{2}(k)} .
$$

From (27), it is obtained that $\Delta \phi(k)$ is

$$
\Delta \boldsymbol{\phi}(k)=-T_{s} \boldsymbol{\Gamma} \sigma(k) \boldsymbol{\theta}(k)-T_{s} \kappa \Gamma \frac{\boldsymbol{\omega}(k) e_{0}(k)}{\bar{m}^{2}(k)},
$$

and as $\boldsymbol{\phi}(k)$ can be written as $\boldsymbol{\phi}(k+1)-\Delta \boldsymbol{\phi}(k)$, follows that

$$
\Delta V(k) \leq 2 \boldsymbol{\phi}^{T}(k+1) \Gamma^{-1} \Delta \boldsymbol{\phi}(k) .
$$

By applying Cauchy-Schwarz Inequality, the fact that $2 \boldsymbol{\phi}^{T}(k) \boldsymbol{\theta}(k)=\|\boldsymbol{\phi}(k)\|^{2}+\|\boldsymbol{\theta}(k)\|^{2}-\left\|\boldsymbol{\theta}^{*}\right\|^{2}$ [36], completing squares and organizing the terms, follows that

$$
\begin{array}{r}
\Delta V(k) \leq \ldots \\
\ldots-T_{s} \boldsymbol{\sigma}(k)\left(\|\boldsymbol{\theta}(k)\|^{2}-\left\|\boldsymbol{\theta}^{*}\right\|^{2}-2 T_{s} \sigma(k)\|\boldsymbol{\Gamma}\|\|\boldsymbol{\theta}(k)\|^{2}\right)-\ldots \\
\ldots T_{s} \kappa \frac{\left[\boldsymbol{\phi}^{T}(k) \boldsymbol{\omega}(k)\right]^{2}}{\bar{m}^{2}(k)}+\ldots \frac{\mu^{2} \eta^{2}(k)}{\bar{m}^{2}(k)}-\ldots \\
\ldots T_{s} \kappa \frac{e_{0}^{2}(k)}{\bar{m}^{2}(k)}\left(1-2 T_{s} \kappa \sigma^{2}(k)\|\boldsymbol{\Gamma}\| \frac{\|\boldsymbol{\omega}(k)\|^{2}}{\bar{m}^{2}(k)}\right) .
\end{array}
$$

From (30), the following controller constraints can be concluded to maintain a stable closed-loop operation,

I) $\quad\left(\|\boldsymbol{\theta}(k)\|^{2}-\left\|\boldsymbol{\theta}^{*}\right\|^{2}-2 T_{s} \boldsymbol{\sigma}(k)\|\boldsymbol{\Gamma}\|\|\boldsymbol{\theta}(k)\|^{2}\right) \geq 0$, for positive defined symmetric $\Gamma$ and $\sigma(k) \in\left[0, \sigma_{0}\right]$, where $0<\sigma_{0}<1$ so that it satisfies the following design constraint $2 T_{s} \boldsymbol{\sigma}(k)\|\boldsymbol{\Gamma}\| \leq\|\boldsymbol{\theta}(k)\|^{2}-\left\|\boldsymbol{\theta}^{*}\right\|^{2}$ and therefore $-T_{s} \boldsymbol{\sigma}(k)\left(\|\boldsymbol{\theta}(k)\|^{2}-\left\|\boldsymbol{\theta}^{*}\right\|^{2}-2 T_{s} \boldsymbol{\sigma}(k)\|\boldsymbol{\Gamma}\|\|\boldsymbol{\theta}(k)\|^{2}\right) \leq 0 ;$

II) $-T_{s} \kappa \frac{\left[\boldsymbol{\phi}^{T}(k) \boldsymbol{\omega}(k)\right]^{2}}{\bar{m}^{2}(k)} \leq 0$ for $T_{s}>0$ and $\kappa>0$;

III) $\left(1-2 T_{s} \kappa|| \boldsymbol{\Gamma} \| \sigma^{2}(k) \frac{\|\boldsymbol{\omega}(k)\|^{2}}{\bar{m}^{2}(k)}\right) \geq 0$, for positive defined symmetric $\Gamma$ and $\sigma(k) \in\left[0, \sigma_{0}\right]$, where $0<\sigma_{0}<1$, and knowing that $\frac{\|\boldsymbol{\omega}(k)\|^{2}}{\bar{m}^{2}(k)}<1$ due to Lemma I, discussed later, so that it satisfies the following design constraint $2 T_{s} \kappa\|\boldsymbol{\Gamma}\| \sigma^{2}(k) \frac{\|\boldsymbol{\omega}(k)\|^{2}}{\bar{m}^{2}(k)} \leq 1, \quad$ and therefore $-T_{S} \kappa \frac{e_{0}^{2}(k)}{\bar{m}^{2}(k)}\left(1-2 T_{s} \kappa \sigma^{2}(k)\|\boldsymbol{\Gamma}\| \frac{\|\boldsymbol{\omega}(k)\|^{2}}{\bar{m}^{2}(k)}\right) \leq 0 ;$

IV) $\frac{\mu^{2} \eta^{2}(k)}{\bar{m}^{2}(k)}$ is limited, small in the mean and belongs to a residual set $\Psi(k)$, as Theorem $\mathrm{I}$, discussed later.

From (30), it can be affirmed that, in presence of unknown dynamics, $\boldsymbol{\phi}(k)$ is limited, once $\Delta V(k)$ is almost always negative. However, when $\Delta V(k)$ is positive, $V(k)$ increases, but $\|\boldsymbol{\phi}(k)\|$ and $\|\boldsymbol{\theta}(k)\|$ also increase, and $\Delta V(k)$ turns negative again. Thus, $V(k)$ has an upper bound given by $\bar{V}(k)=\left[\mu^{2} \eta^{2}(k)\right] / \bar{m}^{2}(k)$.

To prove that all closed-loop signals are limited, consider the Lemma I [36],

Lemma I. Be the system: $\boldsymbol{x}(k+1)=\boldsymbol{A} \boldsymbol{x}(k)+\boldsymbol{B} \bar{U}$, where $\boldsymbol{x}(k) \in \mathfrak{R}^{n}$ are states and the eigenvalues of $\boldsymbol{A}$ satisfy $\left|\boldsymbol{\lambda}_{i}(\boldsymbol{A})\right|<$ $\delta_{0}-\delta_{2}, i=1,2, \ldots, n, 0<\delta_{0}<1,0<\delta_{2}<\delta_{0}<1$, and || $\bar{U}|| \leq|u(k)|+|y(k)|$. Therefore, there is a positive constant $\xi_{1}$ such that $\|x(k)\| / m(k)<\xi_{0}+\varepsilon(k)$, where $\varepsilon(k)$ is a term that depends on initial conditions and converges exponentially to zero when $k \rightarrow \infty$.

Proof of Lemma I: it is presented in [36], and then, here it is omitted.

Using Lemma I, the system closed-loop signals are proven to be limited by majorant signal. As $\bar{m}^{2}(k)=m^{2}(k)+\boldsymbol{\omega}^{T}(k) \boldsymbol{\Gamma} \boldsymbol{\omega}(k)$ and $m(k+1)=$ $\left(1-T_{s} \delta_{0}\right) m(k)+T_{s} \delta_{1}(1+|u(k)|+|y(k)|)$, then the 
boundedness plant output and control action follows directly,

$$
\begin{array}{r}
\frac{|y(k)|}{\bar{m}^{2}(k)}=\ldots \\
\cdots \frac{y(k)}{\left(1-T_{s} \boldsymbol{\delta}_{0}\right) m(k)+T_{s} \boldsymbol{\delta}_{1}(1+|u(k)|+|y(k)|)+\boldsymbol{\omega}^{T}(k) \boldsymbol{\Gamma} \boldsymbol{\omega}(k)},
\end{array}
$$

or,

$$
\frac{|y(k)|}{\bar{m}^{2}(k)} \leq \xi_{1}+\varepsilon(k),
$$

where $\xi_{1}$ is positive constant and $\varepsilon(k)$ is a term that decays exponentially to zero when $k \rightarrow \infty$, and

$$
\frac{|u(k)|}{\bar{m}^{2}(k)}=\ldots
$$

$$
\cdots \frac{u(k)}{\left(1-T_{s} \delta_{0}\right) m(k)+T_{s} \delta_{1}(1+|u(k)|+|y(k)|)+\boldsymbol{\omega}^{T}(k) \boldsymbol{\Gamma} \boldsymbol{\omega}(k)},
$$

or,

$$
\frac{|u(k)|}{\bar{m}^{2}(k)} \leq \xi_{2}+\varepsilon(k)
$$

where $\xi_{2}$ is a positive constant.

Next, to prove $\eta$ limitation, note that $\eta=\Delta(z) u$, where $\Delta(z)$ is a stable and strictly proper transfer function, due to assumption $A 3$ from controller design. Thereby, as $u(k)$ is bounded, then $\eta(k)$ also is bounded,

$$
\frac{|\eta|}{\bar{m}^{2}}=\Delta(z) \frac{u}{\left(1-T_{s} \delta_{0}\right) m+T_{s} \delta_{1}(1+|u|+|y|)+\boldsymbol{\omega}^{T} \boldsymbol{\Gamma} \boldsymbol{\omega}},
$$

or,

$$
\frac{|\eta(k)|}{\bar{m}^{2}(k)} \leq \xi_{3}+\varepsilon(k)
$$

where $\xi_{3}$ is a positive constant.

Besides, to prove $\boldsymbol{\omega}(k)$ boundedness, note that $\boldsymbol{\omega}^{T}(k)=$ [ $\left.u(k) u(k-1) y(k) e_{0}(k-1)\right]$, where the regulation error $e_{0}(k)$ belongs to a residual set $\Psi(k)$, discussed later, and then it is limited, $u(k)$ and $y(k)$ are also bounded, as previously discussed, therefore the $\boldsymbol{\omega}(k)$ boundedness follows directly,

$$
\frac{|\boldsymbol{\omega}(k)|}{\bar{m}^{2}(k)}=\frac{\boldsymbol{\omega}(k)}{\left(1-T_{s} \boldsymbol{\delta}_{0}\right) m+T_{s} \boldsymbol{\delta}_{1}(1+|u|+|y|)+\boldsymbol{\omega}^{T}(k) \boldsymbol{\Gamma} \boldsymbol{\omega}(k)},
$$

or,

$$
\frac{|\boldsymbol{\omega}(k)|}{\bar{m}^{2}(k)} \leq \xi_{4}+\varepsilon(k),
$$

where $\xi_{4}$ is a positive constant.

Finally, the $m(k+1)$ boundedness proof start by dividing both sides of (17) by $\bar{m}^{2}(k)$ and using the fact that $\bar{m}^{2}(k)>$ $m(k+1) \forall k>0$, once $\boldsymbol{\omega}^{T}(k) \boldsymbol{\Gamma} \boldsymbol{\omega}(k) \geq 0$ for positive defined $\boldsymbol{\Gamma}$, and by boundedness of $y(k), u(k)$ and $\boldsymbol{\omega}(k)$, previously discussed, then the proof follows directly,

$$
\begin{array}{r}
\frac{|m(k+1)|}{\bar{m}^{2}(k)}=\ldots \\
\frac{\left(1-T_{s} \delta_{0}\right) m(k)+T_{s} \delta_{1}(1+|u(k)|+|y(k)|)}{\left(1-T_{s} \delta_{0}\right) m(k)+T_{s} \delta_{1}(1+|u(k)|+|y(k)|)+\boldsymbol{\omega}^{T}(k) \boldsymbol{\Gamma} \boldsymbol{\omega}(k)}
\end{array}
$$

or,

$$
\frac{|m(k+1)|}{\bar{m}^{2}(k)} \leq \xi_{5}+\varepsilon(k)
$$

where $\xi_{5}$ is a positive constant.

The controller stability is analysed by Theorem I.

Theorem I. Be $r(k)$ and $\Delta r(k)$ uniformly limited signals. Be also, the plant given by (2)-(4), subject to assumptions $A 1-A 4$, control structure (12), along the adaptation algorithm (15)-(18). Then, there is a $\mu^{*}>0$ such that for all $\mu \in$ $\left[0, \mu^{*}\right)$, all closed-loop signals are limited to any limited initial conditions. Furthermore, the controller stability analysis shows that regulation error converges to the residual set, $\Psi(k)$,

$$
\Psi(k)=\left\{e_{0}(k): \lim _{N \rightarrow \infty} \sup _{N>0} \frac{1}{N} \sum_{i=0} N\left|e_{0}(k)\right| \leq q_{6} \sqrt{\varepsilon_{0}}+\mu q_{7}\right\}
$$

$\forall i>0, N \rightarrow \infty$, where $N$ is the amount of samples in one period, $q_{6}$ and $q_{7}$ are constants and $\varepsilon_{0}>0$ is a small arbitrary number.

Proof of Theorem I: it is similar to the proof presented in [36], and then, here it is omitted.

\section{ACKNOWLEDGEMENTS}

This study was financed in part by the Coordenação de Aperfeiçoamento de Pessoal de Nível Superior - Brasil (CAPES/PROEX)-Finance code 001. The authors would also like to thank the INCT-GD and the finance agencies $(\mathrm{CNPq}$ 465640/2014-1, CNPq Projeto 424997/2016-9, CAPES 23038.000776/2017-54 and FAPERGS 17/2551-0000517-1).

\section{REFERENCES}

[1] P. K. Ndwali, J. G. Njiri, E. M. Wanjiru, "Optimal Operation Control of Microgrid Connected Photovoltaic-Diesel Generator Backup System Under Time of Use Tariff", Journal of Control, Automation and Electrical Systems, pp. 1-14, aug. 2020.

[2] R. Singh, R. C. Bansal, A. R. Singh, R. Naidoo, "Multi-objective optimization of hybrid renewable energy system using reformed electric system cascade analysis for islanding and grid connected modes of operation", IEEE Access, vol. 6, pp. 47332-47354, sep. 2018.

[3] V. Suresh, S. Sreejith, S. K. Sudabattula, V. K. Kamboj, "Demand response-integrated economic dispatch incorporating renewable energy sources using ameliorated dragonfly algorithm", Electrical Engineering, vol. 101, no. 2, pp. 421-442, jun. 2019. 
[4] M. E. Meral, D. Çelik, "Comparison of SRF/PIand STRF/PR-based power controllers for gridtied distributed generation systems", Electrical Engineering, vol. 100, no. 2, pp. 633-643, jun. 2018.

[5] O. Noureldeen, I. Hamdan, "Design of robust intelligent protection technique for large-scale gridconnected wind farm", Protection and Control of Modern Power Systems, vol. 3, no. 1, p. 17, jun. 2018.

[6] S. Padmanaban, F. Blaabjerg, P. Wheeler, J. O. Ojo, A. H. Ertas, "High-voltage dc-dc converter topology for pv energy utilization, investigation and implementation", Electric Power Components and Systems, vol. 45, no. 3, pp. 221-232, dec. 2017.

[7] M. Bajaj, A. K. Singh, "An analytic hierarchy processbased novel approach for benchmarking the power quality performance of grid-integrated renewable energy systems", Electrical Engineering, pp. 1-21, sep. 2020.

[8] E. Ali, S. A. Elazim, A. Abdelaziz, "Optimal allocation and sizing of renewable distributed generation using ant lion optimization algorithm", Electrical Engineering, vol. 100, no. 1, pp. 99-109, mar. 2018.

[9] S. A. O. da Silva, L. B. G. Campanhol, V. D. Bacon, L. P. Sampaio, "Single-phase gridconnected photovoltaic system with active power line conditioning", Eletrônica de Potência, vol. 20, no. 1, pp. 8-18, fev. 2015.

[10] A. Farakhor, M. Abapour, M. Sabahi, "Design, analysis, and implementation of a multiport DC-DC converter for renewable energy applications", IET Power Electronics, vol. 12, no. 3, pp. 465-475, mar. 2018.

[11] B. Sakallioglu, B. Esenboga, T. Demirdelen, M. Tümay, "Performance evaluation of phaseshifting transformer for integration of renewable energy sources", Electrical Engineering, vol. 102, pp. 2025-2039, dec. 2020.

[12] J. Storey, P. R. Wilson, D. Bagnall, "The optimizedstring dynamic photovoltaic array", IEEE Transactions on Power Electronics, vol. 29, no. 4, pp. 1768-1776, apr. 2013.

[13] E. Twining, D. G. Holmes, "Grid current regulation of a three-phase voltage source inverter with an LCL input filter", IEEE Transactions on Power Electronics, vol. 18, no. 3, pp. 888-895, may 2003.

[14] R. Jadeja, A. D. Ved, S. K. Chauhan, T. Trivedi, "A random carrier frequency PWM technique with a narrowband for a grid-connected solar inverter", Electrical Engineering, pp. 1-13, sep. 2020.

[15] D. Li, Z. Zhu, "A novel integrated power quality controller for microgrid", IEEE Transactions on Industrial Electronics, vol. 62, no. 5, pp. 2848-2858, may 2014.

[16] "IEEE Standard for Interconnection and Interoperability of Distributed Energy Resources with Associated Electric Power Systems Interfaces", IEEE Std 1547-2018 (Revision of IEEE Std 15472003), vol. 19, no. 5, pp. 1-138, apr. 2018.
[17] J. Dannehl, F. W. Fuchs, P. B. Thogersen, "PI state space current control of grid-connected PWM converters with LCL filters", IEEE Transactions on Power Electronics, vol. 25, no. 9, pp. 2320-2330, sep. 2010.

[18] R. Teodorescu, F. Blaabjerg, M. Liserre, P. C. Loh, "Proportional-resonant controllers and filters for grid-connected voltage-source converters", IEE Proceedings - Electric Power Applications, vol. 153, no. 5, pp. 750-762, sep. 2006.

[19] J. C. Moreno, J. M. E. Huerta, R. G. Gil, S. A. Gonzalez, "A robust predictive current control for three-phase grid-connected inverters", IEEE Transactions on Industrial Electronics, vol. 56, no. 6, pp. 1993-2004, jun. 2009.

[20] J. Xu, S. Xie, T. Tang, "Active damping-based control for grid-connected $L C L$-filtered inverter with injected grid current feedback only", IEEE Transactions on Industrial Electronics, vol. 61, no. 9, pp. 4746-4758, sep. 2014.

[21] F. Huerta, D. Pizarro, S. Cobreces, F. J. Rodriguez, C. Giron, A. Rodriguez, "LQG servo controller for the current control of LCL grid-connected voltagesource converters", IEEE Transactions on Industrial Electronics, vol. 59, no. 11, pp. 4272-4284, nov. 2012.

[22] L. A. Maccari, J. R. Massing, L. Schuch, C. Rech, H. Pinheiro, R. C. Oliveira, V. F. Montagner, "LMIbased control for grid-connected converters with LCL filters under uncertain parameters", IEEE Transactions on Power Electronics, vol. 29, no. 7, pp. 3776-3785, jul. 2013.

[23] R. P. Vieira, L. T. Martins, J. R. Massing, M. Stefanello, "Sliding mode controller in a multiloop framework for a grid-connected VSI with LCL filter", IEEE Transactions on Industrial Electronics, vol. 65, no. 6, pp. 4714-4723, jun. 2017.

[24] L. A. Maccari Jr, D. M. Lima, G. G. Koch, V. F. Montagner, "Robust Model Predictive Controller Applied to Three-Phase Grid-Connected LCL Filters", Journal of Control, Automation and Electrical Systems, vol. 31, pp. 447-460, apr. 2020.

[25] M. F. Braga, C. F. Morais, L. A. Maccari, E. S. Tognetti, V. F. Montagner, R. C. L. F. Oliveira, P. L. D. Peres, "Robust stability analysis of gridconnected converters based on parameter-dependent lyapunov functions", Journal of Control, Automation and Electrical Systems, vol. 28, no. 2, pp. 159-170, apr. 2017.

[26] G. G. Koch, C. R. Osório, H. Pinheiro, R. C. Oliveira, V. F. Montagner, "Design Procedure Combining Linear Matrix Inequalities and Genetic Algorithm for Robust Control of Grid-Connected Converters", IEEE Transactions on Industry Applications, vol. 56, no. 2, pp. 1896-1906, mar./apr. 2019.

[27] R. V. Tambara, J. R. Massing, H. Pinheiro, H. A. Gründling, "A digital RMRAC controller based on a modified RLS algorithm applied to the control of the output currents of an LCL-filter connected to the grid", in 15th European Conference on Power Electronics and Applications (EPE), pp. 1-8, IEEE, sep. 2013. 
[28] J. R. Massing, M. Stefanello, H. A. Grundling, H. Pinheiro, "Adaptive current control for gridconnected converters with LCL filter", IEEE Transactions on Industrial Electronics, vol. 59, no. 12, pp. 4681-4693, dec. 2011.

[29] R. V. Tambara, J. M. Kanieski, J. R. Massing, M. Stefanello, H. A. Gründling, "A DiscreteTime Robust Adaptive Controller Applied to GridConnected Converters with LCL Filter", Journal of Control, Automation and Electrical Systems, vol. 28, no. 3, pp. 371-379, jun. 2017

[30] P. J. D. d. O. Evald, R. V. Tambara, H. A. Gründling, "A Discrete-Time Robust MRAC Applied on GridSide Current Control of a Grid-Connected Three-Phase Converter with LCL Filter", in ELECTRIMACS 2019, pp. 45-57, Springer, 2020.

[31] M. A. Soliman, H. M. Hasanien, H. Z. Azazi, E. E. El-Kholy, S. A. Mahmoud, "An adaptive fuzzy logic control strategy for performance enhancement of a grid-connected PMSG-based wind turbine", IEEE Transactions on Industrial Informatics, vol. 15, no. 6, pp. 3163-3173, jun. 2019.

[32] A. A. Mohamed, H. Metwally, A. El-Sayed, S. Selem, "Predictive neural network based adaptive controller for grid-connected PV systems supplying pulse-load", Solar Energy, vol. 193, pp. 139-147, nov. 2019.

[33] L. C. Borin, C. R. D. Osório, G. G. Koch, T. S. Gabbi, R. C. L. F. de Oliveira, V. F. Montagner, "Robust control design procedure based on particle swarm optimization and Kharitonov's theorem with an application for PMSMs", Eletrônica de Potência, vol. 25, no. 2, pp. 219-229, jun. 2020.

[34] C. R. D. Osório, G. G. Koch, I. Cleveston, L. C. Borin, F. H. Dupont, R. C. L. F. Oliveira, V. F. M. Montagner, "Otimização multiobjetivo para controle robusto aplicado a inversores conectados à rede", Eletrônica de Potência, vol. 24, no. 1, pp. 107-115, mar. 2018.

[35] W. C. Duesterhoeft, M. W. Schulz, E. Clarke, "Determination of instantaneous currents and voltages by means of alpha, beta, and zero components", Transactions of the American Institute of Electrical Engineers, vol. 70, no. 2, pp. 1248-1255, jul. 1951.

[36] P. Ioannou, K. Tsakalis, "Robust discrete-time adaptive control", in Adaptive and Learning Systems, pp. 73-85, Springer, 1986.

[37] K. Ogata, Discrete-time control systems, vol. 2, Prentice Hall Englewood Cliffs, NJ, 1995.

[38] P. Ioannou, K. Tsakalis, "A robust direct adaptive controller", IEEE Transactions on Automatic Control, vol. 31, no. 11, pp. 1033-1043, nov. 1986.

[39] M. Liserre, F. Blaabjerg, S. Hansen, "Design and control of an LCL-filter-based three-phase active rectifier", IEEE Transactions on Industry Applications, vol. 41, no. 5, pp. 1281-1291, sep./oct. 2005.

[40] R. Cardoso, R. F. de Camargo, H. Pinheiro, H. A. Gründling, "Kalman filter based synchronisation methods", IET Generation, Transmission \& Distribution, vol. 2, no. 4, pp. 542-555, jul. 2008.
[41] P. J. D. O. Evald, R. V. Tambara, H. A. Gründling, "A direct discrete-time reduced order robust model reference adaptive control for grid-tied power converters with LCL filter", Eletrônica de Potência, vol. 25, no. 3, pp. 361-372, jul./sep. 2020.

[42] P. Ioannou, K. Tsakalis, "A robust discrete-time adaptive controller", in 25th Conference on Decision and Control (CDC), pp. 838-843, IEEE, dec. 1986.

\section{BIOGRAPHIES}

Paulo Jefferson Dias de Oliveira Evald received the B.Sc. in Automation Engineering and M. Sc. in Computer Engineering by Federal University of Rio Grande at (FURG), in 2016 and 2018, respectively. He worked as Auxiliary Professor at Federal University of Rio Grande, from 2017 to 2019. Currently, he is Ph.D. candidate in Electrical Engineer at Federal University of Santa Maria (UFSM) and Assistant Professor at Franciscan University (UFN). Besides, he is a researcher at the Power and Control Electronics Group (GEPOC). Also, he is a effective member of the Brazilian Society of Power Electronics (SOBRAEP). Your main research interests include adaptive control theory, renewable energy and power electronics control applications.

Guilherme Vieira Hollweg received the B.Sc. and M. Sc. in Electrical Engineering by UFSM, in 2017 and 2019, respectively. Currently, he is a Ph.D. student in Electrical Engineer at UFSM. Besides, he is a researcher at GEPOC. Your main research interests include adaptive control theory, non-linear control theory, renewable energy and power electronics control applications.

Rodrigo Varella Tambara received the degree of Technician in Electrotechnics by Industrial Technical College of Santa Maria (CTISM) in 2004, B.Sc., M.Sc. and Ph.D. in Electrical Engineer by UFSM in 2008, 2010 and 2014, respectively. He worked as Assistant Professor at University Franciscan Centre (UNIFRA), from 2014 to 2016, and also worked as Adjunct Professor at UFSM, from 2016 to 2018. Currently, he is currently Adjunct Professor at CTISM and Substitute Coordinator of the higher course in Technology in Industrial Electronics. Besides, he is a researcher at GEPOC and at the Research and Development Group on Electrical and Computer Systems (GSEC). Also, he is a effective member of SOBRAEP. Your main research interests include theory and application of control systems, electronic instrumentation and power electronics.

Hilton Abílio Gründling received the B.Sc. in Electronics Engineering by Pontifical University of Rio Grande do Sul (PUCRS) in 1977, M. Sc. in Electrical Engineering from Federal University of Santa Catarina (UFSC) in 1980 and $\mathrm{Ph.D}$. in Electronic and Computer Engineering by Technological Institute of Aeronautics (ITA) in 1995. He worked as Titular Professor at UFSM, from 1980 to 2016. Since 2017, he is Full Professor at UFSM Campus Cachoeira do Sul (UFSM-CS). Besides, he is a researcher at GEPOC. Your main research interests include discrete-time robust adaptive controllers and grid-tied power systems. 